\title{
Kernos
}

Revue internationale et pluridisciplinaire de religion grecque antique

3| 1990

Varia

\section{Les oracles de l'Italie antique : hellénisme et italicité}

Jacqueline Champeaux

\section{(apenEdition}

Journals

\section{Édition électronique}

URL : http://journals.openedition.org/kernos/975

DOI : $10.4000 /$ kernos.975

ISSN : 2034-7871

\section{Éditeur}

Centre international d'étude de la religion grecque antique

\section{Édition imprimée}

Date de publication : 1 janvier 1990

ISSN : 0776-3824

\section{Référence électronique}

Jacqueline Champeaux, «Les oracles de I'Italie antique : hellénisme et italicité », Kernos [En ligne], 3 | 1990, mis en ligne le 19 avril 2011, consulté le 04 mai 2019. URL : http://journals.openedition.org/ kernos/975; DOI : 10.4000/kernos.975 


\section{LES ORACLES DE L'ITALIE ANTIQUE : HELLÉNISME ET ITALICITÉ}

L'Italie est communément tenue pour une terre pauvre en oracles. Patrii sermonis egestas... : la plainte de Lucrèce (I, 832; III, 260) pourrait être reprise par l'historien de la religion romaine. Pauvre en grands oracles, en centres mantiques de prestige, sans doute : le plus célèbre des oracles nationaux, Préneste, "la Delfi d'Italia» ${ }^{1}$, dont nous traiterons tout d'abord, si grand que soit son prestige, n'est pas en mesure de rivaliser avec son homologue hellénique. Mais, outre Préneste et quelques autres centres réputés, il existe, nous chercherons ensuite à le montrer, une poussière d'oracles locaux, disséminés de par toute la péninsule : tous, ou presque, sont de type cléromantique, fondés sur le tirage des sorts et l'écriture. Ce qui a également contribué à les reléguer dans l'ombre : plus ternes, moins spectaculaires que les oracles grecs, ils ignorent la transe et l'enthousiasme - même si le délire de la Pythie n'est plus ce que l'on pensait. Pourtant, l'attitude de l'Italie étrusco-romaine à l'égard du phénomène oraculaire, de ses méthodes, du problème de l'inspiration prophétique apparaît, à la réflexion, moins simple qu'on ne l'a cru d'ordinaire.

Pour les Anciens, déjà, Préneste, avec son sanctuaire de Fortuna Primigenia, était l'oracle italique par excellence, le plus célèbre de la péninsule : clarissumarum sortium, dit Cicéron, siège d'un culte connu, au IIe siècle av. J.-C., jusque dans le monde grec, en Crète, à Délos, et qui fut visité par Prusias et par Carnéade. Pour nous aussi, Préneste joue un rôle de révélateur. Nous disposons, sur elle, d'une documentation exceptionnelle, littéraire (le chapitre que Cicéron lui a consacré dans le De diuinatione, II, 85-87), épigraphique, archéo-

1 Nous nous bornons à renvoyer, pour la bibliographie, le détail des références et l'état des questions, à J. Champeaux, Fortuna. Recherches sur le culte de la Fortune à Rome et dans le monde romain, Rome, 1982-1987 (Coll. de l'École française, 64), I, p. 3-182 (Préneste, Antium); II, p. 225-231 et 307; aux actes des trois Tables rondes sur La divination dans le monde étrusco-italique, in Caesarodunum, Suppl. 52, 54, 56 (1985-1986); ainsi qu'à nos deux articles à paraître dans les MEFR : "Sors oraculi" (carte, inventaire, iconographie des sorts) et Pour une préhistoire des oracles italiques. 
logique ${ }^{2}$. C'est à elle, complétée par d'autres sources, que nous devons, pour l'essentiel, notre connaissance du rituel des "sorts». Sors, dans le texte de Cicéron, est en effet à prendre au sens propre. On sait que le substantif est employé, à toute époque, avec deux sens : l'un, restreint, précis, qui désigne la tablette utilisée pour le tirage au "sort»; l'autre, large et abusif, qui l'étend à toute "réponse» d'un oracle, quel que soit le procédé par lequel elle est obtenue. Preuve, s'il en était besoin, que, pour les Latins, tout oracle est, par définition, de nature cléromantique.

Cicéron, bien informé par la tradition locale (les Praenestinorum monumenta), et qui, manifestement, avait lui-même visité le sanctuaire, rapporte les origines mythiques des sorts. Car l'oracle de Préneste avait un mythe de fondation, fait non moins exceptionnel dans une Italie dont, au contraire de la Grèce, la faible imagination mythique n'a plus à être soulignée. Les sorts, dit-il plus haut, étaient e terra editas (diu., I, 34), «issus de la terre», c'est-à-dire d'un rocher qu'un notable de la ville, averti par des songes, avait entaillé, et il en était sorti des tablettes de chêne, toutes gravées, insculptas priscarum litterarum notis. Formule qui a pu faire croire qu'ils ne portaient pas chacun une phrase complète, mais une seule lettre, autrement dit qu'ils appartenaient à la catégorie des "oracles alphabétiques" (Buchstabenorakel) ${ }^{3}$. On en doutera, car, s'il s'agit d'un type d'oracle bien attesté dans la mantique grecque, et d'ailleurs non en Grèce propre, mais en Asie mineure, on n'en connaît pas d'exemple en Italie. Il suppose en outre une table de concordance dont on n'a pas trace à Préneste. Il est probable que priscarum litterarum notis signifie simplement que les sorts étaient

2 Les fouilles du sanctuaire ont, depuis la dernière guerre, dégagé un ensemble architectural unique dans l'Italie d'aujourd'hui. Mais les énigmes posées par le culte sont en proportion de sa célébrité : le sens de l'épiclèse Primigenia et la relation de Fortuna à Jupiter (divinité «Primordiale», et non fille «premièrenée» du dieu), la topographie du ou des sanctuaires sont les questions les plus délicates qu'il continue de soulever. Nous croyons avoir, autant qu'il était possible, contribué à les élucider; mais, dans un domaine aussi complexe, rien n'est jamais acquis, et les débats archéologiques les plus récents sur la distribution et l'identification des lieux de culte ont encore obscurci le probleme plus qu'ils ne l'ont éclairci. Cf. F. ZEVI, Oracoli alfabetici, Praeneste e Cuma, in AПAPXAI. Nuove ricerche e studi sulla Magna Grecia e la Sicilia antica in on. di P.E. Arias, Pise, 1982, II, p. 605-609; plus généralement, la dissertation de F. HEINEVETTER, Würfel-und Buchstabenorakel in Griechenland und Kleinasien, Breslau, 1912; M. GuarduccI, Epigrafia greca, Rome, 1967-1978, IV, p. 74-122, dont la synthèse sur les «Oracoli» intéresse aussi bien le domaine latin (également $I, p$. 229 sq. et 460 sq.). 
inscrits "en caractères de l'alphabet ancien", ce qui ne surprendra pas dans la ville qui a livré la plus ancienne des inscriptions latines connues, la célèbre fibule, du second quart du VIIe siècle 4 .

Quant au rituel, les sorts étaient déposés dans un coffre, arca, en bois d'olivier, d'origine prodigieuse lui aussi. Ils y étaient mêlés, miscere, puis en étaient tirés par la main d'un enfant, tollere, ducere, qui sont les termes techniques (cf. CIL, V,5801), de même que les Grecs disent óveî̉ev ó Azós. Deux séries de documents complètent le témoignage de Cicéron. Ce sont, d'abord, les inscriptions locales. Elles ne nous éclairent ni sur les questions posées, ni sur les réponses données par la déesse, mais sur les sacerdoces et même le personnel subalterne du temple (sacristains, entrepreneur attitré). Le personnel sacerdotal se composait, sous l'Empire en tout cas - mais en avait-il toujours été ainsi ? -, de deux prêtres : le sacerdos en titre, et un sortilegus spécialisé, le même prêtre ne pouvant suffire à tout, actes sacrificiels, administration d'un grand sanctuaire et service de l'oracle. D'où le sortilegus, spécialement chargé de présider au tirage des sorts et de les interpréter, qui s'explique par l'importance du temple et l'affluence des consultants.

Nous nous représentons mieux la liturgie des consultations, d'autre part, grâce à deux monuments figurés qui nous la montrent "en action". Un denier de M. Plaetorius Cestianus, datable vers 69-66, et qui se rapporte très certainement à Préneste, montre une petite fille qui présente à deux mains une tablette sur laquelle est inscrit le mot sors ${ }^{5}$ : le puer mentionné par Cicéron pouvait donc aussi être une puella, comme on en a d'autres exemples dans la liturgie romaine. Plus riche encore d'enseignements est un relief votif d'Ostie, dédié, au Ier siècle av. J.-C., par un haruspice - ce qui en garantit l'exactitude religieuse -, à un Hercule Victor oraculaire lui aussi, et dont le rituel imite visiblement celui de Préneste ${ }^{6}$. La partie centrale de ce triptyque montre une grande arca, posée sur un socle, dans le temple même, puisqu'elle fait face à la statue cultuelle du dieu. Un jeune garçon, également, en tire

4 Dont, malgré les doutes récemment élevés à son encontre, en particulier par $M$. GUARDUCCI, nous ne récusons pas l'authenticité. $\mathrm{Cf}$., dans le nouveau supplément au $C I L, \mathrm{I}^{2}$, fasc. IV, 1986, la mise au point d'A. DEGRAssi et H. KRUMMREY, n $^{\circ} 3$, p. 855 sq.

5 SYDENHAM, 801-802; CRAWFORD, 405, 2.

6 G. BECATTI, $I l$ culto di Ercole ad Ostia ed un nuovo rilievo votivo; et Nuovo documento del culto di Ercole ad Ostia, in BCAR, 67 (1939), p. 37-60; 70 (1942), p. 115-125. Dernièrement, R. Chevallier, Ostie antique, ville et port, Paris, 1986, p. 192 et 225-227. 
une tablette qu'il remet au dieu, qui fait lui-même fonction de sortilegus. La scène de droite - celle de gauche est malheureusement tronquée - représente l'aition du culte : des pêcheurs ramènent dans leur filet la statue du dieu et l'arca des sorts. Ajoutons qu'une source était en relation avec le temple. Préneste est un oracle de la terre, Ostie, un oracle des eaux - la Terre et les eaux qui sont les deux sources par excellence de la révélation oraculaire et que nous retrouvons dans d'autres oracles de l'Italie.

Trois descriptions littéraires du tirage des sorts, successivement d'époque républicaine, augustéenne et impériale, complètent ce tableau et concordent avec lui. Il s'agit de la Casina de Plaute (v. 296-418),

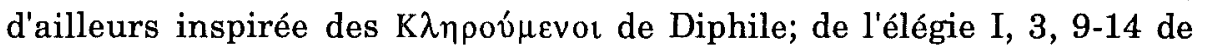
Tibulle, qui décrit une scène de la rue, à Rome, où Délie consulte par l'entremise d'un jeune garçon; et des Métamorphoses d'Apulée, IX, 8, qui met en scène les prêtres charlatans de la Dea Syria. Toutes trois ressortissent à la divination privée, imitation de la liturgie officielle des temples; et divers autres sortilegi privés sont mentionnés par les inscriptions ${ }^{7}$. L'enfant qui tire les sorts à Préneste, à Ostie et chez Tibulle ne devait pas, à notre sens, être présent dans tous les cultes oraculaires : même s'il est, d'une façon générale, le médiateur privilégié des opérations divinatoires et des loteries, nous y verrions plutôt, dans ces deux derniers cas, une imitation du modèle prénestin, qui s'est imposé à tout le Latium comme le type de l'oracle par excellence. De même, la distinction entre le sacerdos et un sortilegus spécialisé doit être une particularité de la divination prénestine : dans les oracles de moindre importance, le même personnage cumulait sans doute les deux fonctions. Mais nous comprenons, grâce à la parodie d'Apulée - les multiples sorts que font tirer ses devins ne portent tous qu'un seul et même texte - combien fondamental était le rôle du sortilegus : il expliquait au consultant en quoi la formule, nécessairement générale, inscrite sur la tablette, répondait à la question particulière qu'il avait posée. Son rôle était celui d'un interprète, c'està-dire, comme les "prophètes" de Delphes, d'un exégète du vouloir divin.

Outre Préneste et la divination oraculaire qui gravite autour d'elle l'Hercule d'Ostie, les diseurs de bonne aventure de Rome -, on discerne une poussière d'oracles locaux, dispersés dans toutes les parties de la péninsule. La simple vue d'une carte oraculaire de l'Italie, que nous avons dressée par ailleurs, dissipe l'illusion que nous évoquions ci-

7 Cf. notre relevé de Caesarodunum, Suppl. 54 (1986), p. 90-113. 
dessus : nous y recensons, toutes époques confondues, une vingtaine d'oracles étrusco-italiques (sans compter les oracles grecs). Précisons qu'aucun d'eux ne se trouve à Rome ${ }^{8}$, qui n'a jamais connu que les devins de carrefour décrits par Tibulle. Ces oracles de l'Italie profonde nous sont connus par des sources soit littéraires, soit archéologiques. Parmi les premières, les historiens jouent un rôle privilégié : Tite-Live, bien sûr, mais aussi les biographes impériaux, Suétone, l'Histoire Auguste. Ce qui n'est pas sans conséquence. L'oracle local est mentionné à l'occasion d'une visite impériale, qui relève du tourisme religieux plus que de la piété à proprement parler. L'auguste visiteur, de passage dans la petite ville, en profite pour interroger l'oracle du lieu, comme, touristes consciencieux, nous ne manquons pas de visiter cathédrales et abbayes. Mais tel n'était pas le but de sa venue, et ces consultations impériales ne sauraient être assimilées à des pèlerinages. Le résultat, c'est que toutes ces attestations sont tardives : elles vont du Ier au IIIe siècle ap. J.-C., alors que, en fait, les oracles consultés remontent certainement à une époque beaucoup plus ancienne, républicaine à coup sûr, si ce n'est même archaïque.

Après celui de Préneste, le second, semble-t-il, en réputation de ces oracles italiques, est celui d'une autre ou plutôt d'autres Fortunes, les deux déesses d'Antium. Le rite de consultation, décrit par Macrobe (Sat., I, 23, 13) au IVe siècle ap. J.-C., était fondé sur les mouvements "spontanés" des statues divines, portées en procession. C'est un rite bien attesté dans les sanctuaires d'Orient, en Syrie, à Héliopolis, Hiérapolis, en Égypte, au temple d'Ammon à l'oasis de Siwa. Non pas, comme on l'a cru, rite tardivement importé en Italie avec l'invasion des religions orientales et qui, en outre, se serait combiné avec la cléromancie. Mais ce rite, comme nous l'avons montré, se suffit à lui-même et y fut introduit, dès l'époque archaïque, par l'intermédiaire de Carthage, avec qui Antium, ville de marins et de pirates, était en relation dès l'époque royale : de fait, elle est nommée dans le premier traité conclu entre Rome et Carthage à la fin du VIe siècle, traité cité par Polybe (III, 22) et dont, aujourd'hui, on ne met plus en doute l'authenticité.

Antium, largement ouverte sur le monde méditerranéen, précocement en contact, d'ailleurs indirect, avec la divination orientale, est un cas unique en Italie. Les autres oracles que nous avons recensés rentrent pour ainsi dire tous dans le moule commun : ce sont des oracles par les sorts. En rayonnant à partir du Latium, nous

8 Malgré l'hypothèse, non confirmée, de M. GuARDucci, que la Junon Moneta du Capitole aurait donné des kavertissements» oraculaires. 
dénombrons ainsi ${ }^{9} \mathrm{l}^{\prime}$ Hercule Victor de Tibur, maître d'un des plus prestigieux sanctuaires italiques; Faléries et diverses villes d'Étrurie (Caere, Punta della Vipera, Viterbe, Arezzo); l'Ombrie, avec le Jupiter Appenninus d'Iguvium et la source du Clitumne décrite par Pline le Jeune (epist., VIII, 8) - oracles l'un de la terre, l'autre des eaux -; au nord, dans la Celtique du Pô, l'oracle de Forum Nouum, près de Parme, et chez les Vénètes, près de Padoue, l'énigmatique oracle de Géryon ${ }^{10}$; au sud, chez les Osques, trois oracles, sur la côte frentanienne et au centre du Samnium : nous trouvons, toujours et partout, de ces sanctuaires à oracles, identifiés, parfois, grâce à une seule sors, découverte par la fouille. Deux de ces pièces sont particulièrement célèbres : le disque de bronze de Cumes, au nom d'Héra, et le caillou du musée de Fiesole, aux noms de Fortuna et de Servius Tullius, dont, malgré les tentatives de M. Guarducci et d'A. Degrassi, la provenance reste incertaine.

Tous ces oracles ont un dénominateur commun : leur nature cléromantique. Mais, pour tout le reste, c'est leur diversité qui frappe. Diversité des dieux qui les dispensent : Fortuna, Hercule, Menerva (à Punta della Vipera), Géryon, etc. Il n'y a pas, en Italie, de grande divinité oraculaire qui se soit imposée comme la puissance mantique par excellence, à l'égal de l'Apollon grec. Sur ce point comme sur d'autres - nous songeons aux cultes thérapeutiques -, l'Italie fait confiance, chaque fois, à ses divinités locales; d'où l'obscurité où restent, pour nous, ces oracles. Non moins grande est la diversité de matière et de forme des sorts qui nous sont parvenus ou que nous voyons décrits ou représentés. Ceux de l'époque classique sont des tablettes de bronze ou de bois, support normal de l'écriture. Mais les fouilles ont livré deux autres formes, beaucoup plus intéressantes. Certains de ces sorts sont des cailloux; d'autres, des disques de bronze ou de plomb ${ }^{11}$,

9 Cf., pour les références, dans Caesarodunum, Suppl. 54 et 56 (1986), l'article d'A. MAGGIANI et le nôtre; ainsi que, dans le présent volume, la communication de D. BRIQUEL.

10 Géryon est l'interpretatio Graeca d'un dieu local Tricéphale, sans doute vénète (plutôt que celtique). Situé dans la zone de sources thermales d'Abano et Montegrotto Terme, où a été mis à jour le très riche dépôt votif d'un sanctuaire des eaux paléovénète, l'oracle de Géryon appartient sans doute aux couches les plus anciennes de la religion italique. Faut-il lui attribuer la série de dix-sept sorts de bronze trouvés au XVIe siècle à "Bahareno" $\left(C I L, \mathrm{I}^{2}, 2173-2189\right)$ ?

11 Certains de ces sorts sont percés d'un trou, les disques en leur centre, les tablettes à leur extrémité; ce qui permettait d'y passer un fil et de les «tirer». L'expression technique sors excidit ne signifie pas que la sors soit «tombée», par exemple sur le sol : elle indique seulement qu'elle est "sortie" au tirage, et l'on 
qui en sont la reproduction artificielle. Quant aux trois sorts cisalpins découverts près de Parme, ce sont des baguettes de bronze, elles aussi reproduction des baguettes de bois qui tiennent une place considérable dans la divination des Celtes, continentaux et insulaires. Nous retrouvons là la dualité grecque des $\kappa \lambda \hat{n}$ por et des $\psi \hat{\eta} \varphi \circ$, les uns, au moins à l'origine, baguettes de bois que, chez Homère, chaque guerrier marque de son signe (Iliade, VII, 171-191), les autres, simples cailloux.

Antium mise à part, la cléromancie domine donc d'une façon écrasante en Italie. Il y a cependant quelques retouches à apporter à ce paysage oraculaire. Nous n'insisterons pas sur l'oracle étrusque de Téthys, peut-être fabuleux, mentionné par Plutarque (Rom., 2,4) et dont traite D. Briquel. Deux oracles italiques, disparus à l'époque classique, remontent à un passé extrêmement ancien : celui que le pic, oiseau sacré de Mars, le picus Martius, rendait à Tiora Matiene en Sabine où, dit Denys d'Halicarnasse ( $I, 14,5)$, perché sur une colonne de bois, il émettait des cris comme les colombes sur le chêne de Dodone. Comparaison, et non pas imitation. Car le pic est bien connu en Italie pour sa sacralité et sa haute valeur divinatoire, et point n'est besoin d'alléguer, pour l'expliquer, une influence de Dodone. De même pour l'oracle de Faunus, mis en scène par Virgile (Aen., VII, 81-95) : son propre fils, le roi Latinus, lui sacrifie et reçoit son oracle étendu à terre, couché sur la peau des victimes. C'est le seul oracle par incubation de l'Italie, dont l'existence a été mise en doute pour la raison majeure qu'il ressemble singulièrement à un autre oracle, mais de l'Italie grecque, celui des héros Calchas et Poladire au mont Drion, dans le massif du Gargano (Strabon, VI, 3, 9).

Ce recensement amène à poser deux questions fondamentales, qui n'en font peut-être qu'une : l'une concerne le rapport des oracles italiques aux oracles grecs; l'autre, leur rapport à la divination inspirée. Y a-t-il, pour l'oracle de Faunus, dérivation d'un modèle grec, ou seulement parallélisme? Nous opterons sans hésiter pour la seconde solution : l'incubation est trop bien attestée dans les autres parties du domaine indo-européen, chez les Celtes, les Germains, en Inde, pour que l'on conclue à l'imitation. C'est un rite extrêmement archaïque que Grecs, d'une part, Latins, de l'autre, ont reçu de leur commun héritage indo-européen. Sur le second point, la divination inspirée, le contraste de l'Italie et de la Grèce est total. Refus de la divination inspirée, monopole quasi exclusif de l'écriture : telle est, par rapport à la Grèce, la

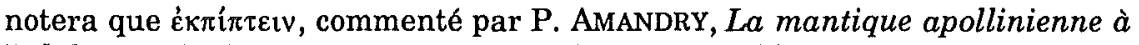
Delphes, Paris, 1950, p. 30, n. 3, a une valeur comparable. 
spécificité italique. On observe, en Italie, des phénomènes de rejet, mais qui ne sont pas si simples. Rejet de l'étranger: Rome se refuse à consulter officiellement la toute proche - trop proche - Préneste ${ }^{12}$ (à moins de quarante kilomètres). Mais elle est fascinée par Delphes que des ambassades officielles vont consulter dans les grandes crises de son histoire : crise dynastique des Tarquins, qui aboutit à l'expulsion des rois; conflit romano-véien; guerre samnite; et, surtout, consultations réitérées de la seconde guerre punique ${ }^{13}$. Il n'y a nulle raison de mettre en doute l'historicité des deux premières de ces consultations, une fois dégagées des éléments folkloriques qui encombrent la fable de Brutus : Rome n'agit pas autrement que les cités étrusques, Caere et Spina, qui avaient leur trésor à Delphes ${ }^{14}$. Fascination qui inspire aussi bien les textes littéraires, Valère-Maxime, Lucain, qui peignent le délire mortel de la Pythie, et même Virgile, dont la Sibylle cumaine a tant de rapports avec la prophétesse de Delphes ${ }^{15}$.

Il existe, de fait, deux personnages types de la divination oraculaire italique : le sortilegus et le uates. Le premier, étymologiquement, n'est pas celui qui lit, mais qui uramasse» les sorts, au sens ancien de legere (et de $\lambda \varepsilon ́ \gamma \varepsilon \iota v)$. Ainsi opéraient les devins italiques, comme les Germains (Tacite, Germ., X, 1-2), comme les Scythes (Hérodote, IV, 67), c'est-à-dire comme leurs ancêtres indo-européens. Vates n'a pas une origine moins lointaine; le mot existe en gaulois, mais le latin, bien sûr, ne le lui a pas emprunté (quoi qu'on en ait dit). En fait, le uates

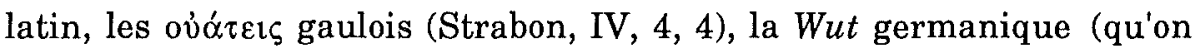
retrouve dans le nom de Wotan, le dieu de l'extase), tous ces mots désignent «linspiré», le prophète qui partage son délire avec le poète. À l'époque classique, le uates n'est plus que le poète, ou le charlatan que les foules crédules consultent, en particulier dans les périodes de crise,

12 Le sénat interdit au consul de 241, Q. Lutatius Cerco, de consulter ces auspices «étrangers" (VAL. MAX., I, 3, 2).

13 Tite-Live, I, 56, 4-12 (Tarquin); V, 15, 3 et 12; 16, 8-11; 23, 1; 25, 10; 28, 1-5 (guerre de Véies et offrande d'un cratère d'or, qui fut déposé dans le trésor des Massaliotes : DIOD., XIV, 93, 3-5; APPIEN, Ital., 8); XXII, 57, 5 et XXIII, 11, 1-6 (après la défaite de Cannes); XXIX, 10, 6 (après la victoire du Métaure); XXIX, 11, 5-6 (avant l'introduction de la Magna Mater); PLINE L'ANCIEN, Histoire naturelle, XXXIV, 26 (guerre samnite). Cf. H.W. PARKE-D.E.W. WORMELL, The Delphic oracle, Oxford, 1956, I, p. 142 sq. et 265-282.

14 STRAB., V, 1, 7 et 2, 3; IX, 3, 8; PLINE L'ANCIEN, Histoire naturelle, III, 120. Cf. la communication de $\mathrm{D}$. BRIQUEL.

15 VaL. MAX., I, 8, 10; LuCAIN, V, 64-236; également STACE, Thébaïde, III, 611-615. Sur la Sibylle, modèle ou reflet de la Pythie, P. AMANDRY, op. cit., p. 21 et 239. 
rendues vulnérables par la défaite; ainsi les sacrificuli ac uates de TiteLive ${ }^{16}$.

Telles sont les deux tentations de la mantique oraculaire romaine, attirée par cela même qu'elle a rejeté : la divination inspirée, sous la double forme du recours sauveur à Delphes et de l'abandon aux séductions douteuses de la divination privée. Mais des figures très anciennes de la religion latine, Albunea, qui deviendra la Sibylle de Tibur, Carmentis qui, étymologiquement, dispense le carmen incantatoire, Faunus, surnommé Fatuus, qui «parle» dans les forêts, et sa parèdre Fauna, suggèrent que ces "prophètes» de l'époque classique ne sont que les héritiers dégénérés de uates primitifs dont le statut social, le prestige mythique en tout cas étaient infiniment plus relevés.

Une Italie pauvre en oracles, rares et tardifs, pâle imitation des oracles grecs? Nous nous sommes efforcé de rectifier, de raviver cette image traditionnelle et négative. L'Italie a eu beaucoup d'oracles, mais de rayonnement purement local. Nous les découvrons au hasard d'une fouille : leur liste n'est donc certainement pas close et a chance de s'accroître à la faveur de nouvelles découvertes. Une Italie qui ne connaissait pas la divination inspirée - ou qui ne la connaissait plus ? Qui l'avait volontairement écartée de sa religion officielle, comme elle avait volontairement oublié sa mythologie ? Les deux choix relèvent sans doute de la même attitude psychologique. Mais, si l'on essaie de remonter au delà, l'Italie archaïque a sans doute connu et pratiqué toutes les ressources de l'art divinatoire : divination par les signes, ceux qu'observent l'augure romain ou l'haruspice étrusque; divination par l'écoute de la parole divine, qu'elle soit transcrite par l'écriture, ou directement énoncée par des uates inspirés et des divinités qui, ellesmêmes, dispensent la "parole" ou l'«incantation" sacrée. La divination italique en général, et oraculaire en particulier, est à la fois plus riche, plus complexe et, tout en préservant l'indépendance d'une ombrageuse italicité, plus proche des canons grecs qu'on ne le soupçonnait.

Université de Rennes II

Jacqueline CHAMPEAUX

Avenue Gaston Berger, 6

F - 35043 RENNES Cedex

$16 \mathrm{XXV}, 1,8$; cf. XXXV, 48, 13; XXXIX, 8, 3 et 16, 8. 